pain, stouter, and looked and felt better. Figs. 1 and 2 show very well the condition before and after the operation.

Remarks by Mr. Brook. - Gluteal aneurysm is very rare, but the interest of this case is further enhanced by the presence of the fractured ilium, produced, of course, by the same blow which ruptured the artery. The absence of repair was remarkable and the appearance and impression it gave to the touch were those of a fracture of a few hours. This could only be accounted for by the continual swirl of the blood at very high pressure over the fractured surfaces. No doubt union had taken place by buttress formation on the inner surface of the bone. Apart from rest and dietary the choice of treatment of gluteal aneurysm lies between : (1) the introduction of needles with or without electrolysis; (2) ligature of the internal iliac artery; (3) the operation of Antyllus; and (4) the operation of Antyllus aided by intraabdominal compression of the internal iliac. It was decided

FiG. 2.

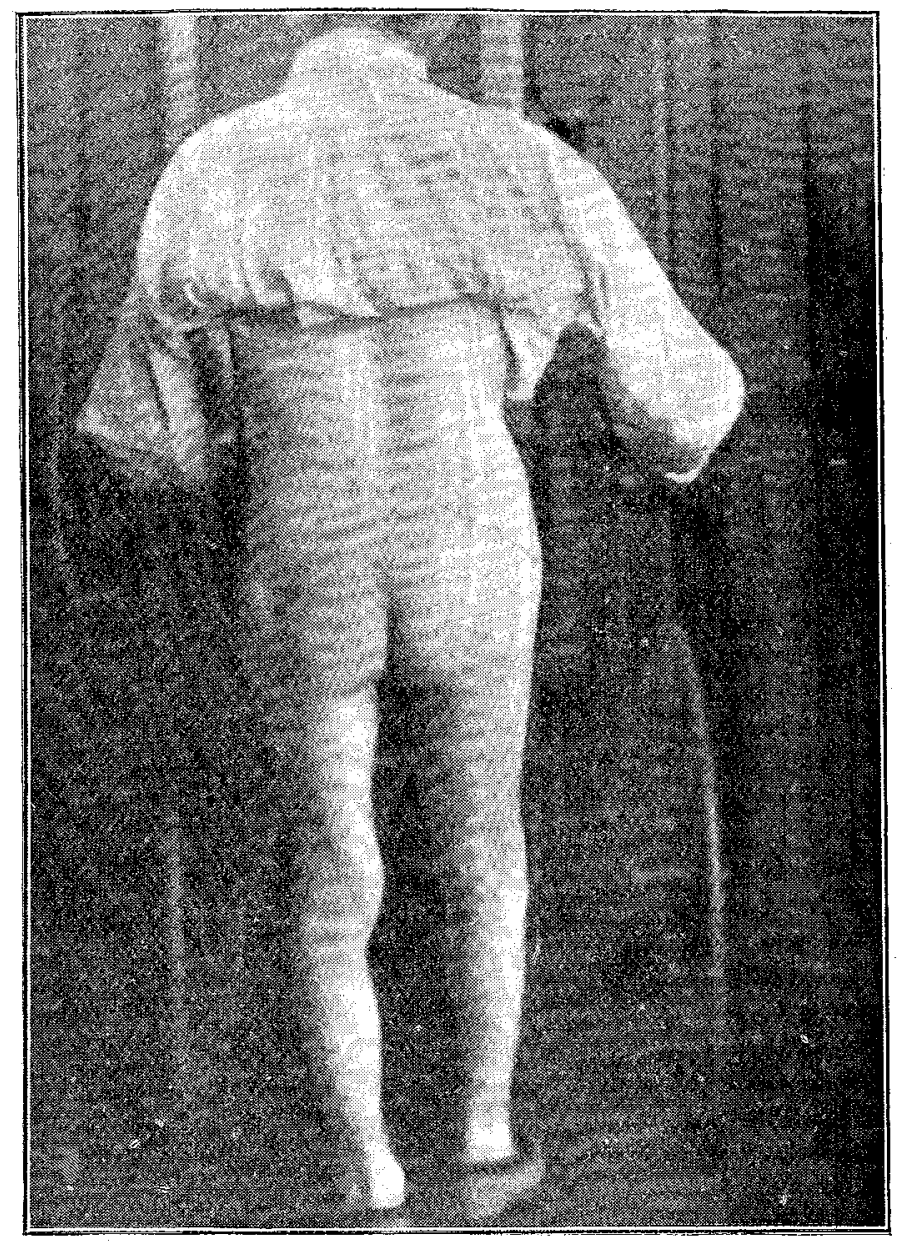

Condition after the operation.

to give treatment by rest, dietary, and iodide of potassium a trial because the tumour was very firm, incompressible, and non-fluctuant and the pulsation not marked, giving the impression that the cavity was nearly obliterated by clot. From the disappearance of the pulsation and bruit it looked at first as though this would be successful. But although the circulation through the distal part of the artery eviclently became arrested the subsecuent increase in size and the altered appearance of the skin showed that the latter would undoubtedly slough unless further measures were promptly adopted. The danger of setting up suppuration in the sac by the use of needles seemed likely to be accentuated by the devitalised condition of the skin and subcutaneous tissues and the choice of treatment came to lie between ligature of the internal iliac and the old operation of Antyllus, aided or not by intraperitoneal compression of the artery. The simplicity and safety of the procedure adopted led me to select it and its ease of performance justified $m y$ anticipations in this respect. Both Syme and at the beginning of the last century Bell opened up the sac in gluteal aneurysm and successfully tied the artery without the assistance of any compression, but, as Jacobson ${ }^{1}$ points out,

I Jacobson and Stewart: Operative Surgery, fourth edition, vol, ii., p. 26 . there is always a risk of fatal hæmorrhage from the artery having retracted into the pelvis. The limited suppuration which occurred was due to the impossibility of obliterating the cavity after operation by means of compressing its walls, being largely composed of muscle tissue rigid with infiltration. The wound was soundly healed before the patient left the hospital, neither of the celluloid ligatures having appeared. The credit of having been the first to devise and to perform this operation belongs to my friend. Mr. W. H. Battle who in 1898 published a case ${ }^{2}$ which he successfully treated in this way at the Royal Free Hospital. So far as I know this is the only case on record. I take this opportunity of acknowledging my indebtedness to my colleague, Mr. J. K. Couch, who kindly assisted by compressing the artery, and also to Mr. F. P. Edwards, assistant house surgeon, who, in the temporary absence of Dr. Muir, assisted at the operation and for the first few days helped in the conduct of the after. treatment.

\section{THE RELATION OF THE THYMUS GLAND TO MARASMUS.}

BY JOHN RUHRÄH, M.D.,

CLINICAT PROFESSOR OF PEDIATRICS, COLLEGE OF PHYSICIANS AND SURGEONS, BALTIMORE, MARYLAND, U.S.A.

THE pathology of infant life has been to a certain extent a very much neglected field and I feel positive that a rich harvest awaits the workers who will be painstaking enough to study both in the ward and in the dead-house. In most places the necropsies on infants are hastily done and the record frequently made in the report, "Nothing abnormal noted." As a matter of actual fact, regarding many organs during infancy there are few who know normal from abnormal or who can tell from a macroscopical examination what the microscope will reveal. There are, I am sure, clinical entities with well-marked lesions which are waiting to be described. As is known, one frequently sees an infant sicken and die without diagnosis during life and no adequate explanation after the necropsy has been made. Many of these cases come under the head of marasmus and it was with the intention of trying to throw some light on this interesting condition that especial care was taken with our necropsies on infants. The following brief report, which I hope will stimulate others to undertake similar studies, is based on about 85 necropsies. The first 23 have been carefully tabulated by my colleagues, Dr. Stokes and Dr. Rohrer and myself, and while the remainder have not been tabrlated they have been studied and the conclusions reached from the first 23 remain unchanged.

It is a curious fact that the most characteristic lesion of marasmus, excepting the wasting of fat and muscles, while known for many years, is not mentioned in any of the textbooks on the diseases of children. This lesion is a wasting of the thymus gland which will be described later. Parrot, in 1877, called attention to the disease which he called athrepsia, the so-called wasting disease of infants, known also as marasmus, simple atrophy, and atrophia primitiva infantum. The term inanition, also used, should be restricted to those acute cases of starvation which have a typical course and characteristic symptoms. There are two conditions in which we see atrophy in infants-the primary cases, where the cause is as yet unknown, and the second. ary cases following definite pathological conditions. The dividing line cannot at this time be definitely drawn. All cases occurring in the course of the easily recognised diseases may at once be placed in the group of secondary casesthose following tuberculosis or syphilis, for example. Clinically most cases are seen in infants which have had improper food and care. Some authors would also place these in the list of secondary cases. They would place under the head of primary atrophies only those where the assimilation was at fault or, in other words, those cases receiving proper care and a physiologically correct diet. This is, we think, a division practically useless; and for the present until we have definite information the cases should be divided, on a pathological basis, into those were there are lesions of definite diseases and those where there are no special and constant lesions except the wasting of the

3 A paper read before the British Medical Association at Swansea on July $29 \mathrm{th}, 1903$. 
muscles and the body fat, and, as we shall see, of the thymus gland as well.

The more important contributions to the pathology of the condition are as follows. Baginsky thought that the disease was due to an atrophy of the mucous membrane of the intestine and that it was owing to this condition that absorption was intefered with and hence the atrophy. $\mathrm{He}$ estimated the amount of nitrogen ingested and that excreted and found that the amount of nitrogen absorbed in these atrophic infants was 50 per cent. below the normal. Similar anatomical studies were made by Fenwick. Since that time many investigators have disproved this anatomical statement. In the majority of cases the changes observed were due to the stretching of the intestinal wall by distension with intestinal gases and also partly in many cases due to post-mortem changes. Bendix found that Baginsky's estimate concerning the nitrogen was exaggerated; and Heubner and Rubner, as the result or experiments carried out in a somewhat different manner, formulated the idea that there was sufficient food taken and absorbed but that the trouble was due to the auto-intoxication from the decomposition of food in the intestinal tract. Czerny is of the same opinion but holds that the intoxication is of an acid nature. The French writers regard the condition as an infection or an intoxication due to a subacute or a chronic infection of the intestinal tract. Marfan has more recently stated that the condition is due to a lack of or a lessened activity of the nutritive ferments, this in turn being due to the intestinal infection. We may safely assert, I think, that the trouble is not due to the amount of food ingested or to the amount absorbed from the intestinal tract, but that it is due to the mal-assimilation of the food material in the body. Whether the thymus gland has anything to do with assimilation or not we are not prepared to say, but it is certain that the thymus gland bears a direct relation to the state of the nutrition of the body. It increases in size until about the second year, when it remains stationary, growing relatively smaller when compared to the size of the growing kody. At puberty, when the body growth is largely over, it begins to atrophy and at about 25 years of age, when the body has attained its full growth, it is gradually replaced by fatty and connective tissue. Herard, at a subsequent date Friedleben and more recently Mettenheimer, have pointed out the relation of the size of the thymus to the state of nutrition of the body. Of this this there can be no doubt, as it rests on a firm basis of trustworthy observation.

The function of the thymus is as yet unknown. The gland weighs about 12 grammes at birth. Histologically it consists of a connective tissue framework holding up a parenchyma consisting of a cortex and medulla which are similar in structure, but the cortex contains normally many more cells than the medulla. The parenchyma consists of a network of endothelial cells resembling the reticular spaces of the lymphatic structures. This space is filled with lymphoid cells and also with a few neutrophilic and eosinophilic leucocytes and with a few giant cells. Here and there in the structure are little islands of epithelial cells with a peculiar concentric arrangement not unlike that found in the concentric bodies of an epithelium. These are the so-called Hassall bodies or corpuscles. They are the remains of the epithelium which forms the principal part of the thymus gland during embryological development.

Our studies cover 18 cases of marasmus. The average weight of the gland was 2.2 grammes. Excepting the ter minal infection the atrophy of the thymus was the only lesion found in any of the necropsies. The changes in the gland consisted in a great atrophy. Histologically the fibrous capsule of the gland is thicker than normal and the trabeculæ are also greatly thickened and the increased interlobular tissue frequently cuts the lobules up into irregular masses. Sometimes there is more fibrous tissue present than there is lymphoid structure. In well-marked cases there is more reticular tissue present than lymphocytes and leucocytes. In most of the cases there was an increase in size and a hyaline degeneration of the Hassall bodies. The state of the nutrition of infants may be estimated by a microscopical examination of the thymus. In those of normal infants the structure of the thymus is unaltered and the differentiation between cortex and medulla is plainly visible. In moderate atrophy the cortex and medulla are not easily distinguished, while in severe atrophies the changes mentioned above are plainly seen. In secondary atrophy the change is one of degree and we found in our cases the average weight of the thymus to be 3.41 grammes. We tried the administration of the thymus gland tablets in cases of marasmus and as far as could be determined there were no changes produced by the use of the gland in the dried form. We arrived at the following conclusions: (1) atrophy of the thymus gland is always found in cases of infantile atrophy; (2) the condition of the thymus is an index of the general nutrition of the infant; and (3) the state of the nutrition in infants may be estimated by a microscopic examination of the thymus at the necropsy.

\section{A SERIES OF CASES OF PERFORATING GASTRIC AND DUODENAL ULCERS.}

\author{
By B. G. A. MOYNIHAN, M.S. LoND., F.R.C.S. ENG., \\ ASSISTANT SURGEON TO THE LEEDS GENERAL INFIRMARY.
}

THE perforation of a gastric or duodenal ulcer is one of the most serious and most overwhelming catastrophes that can befall a human being. The onset of the symptoms is sudden, the course is rapid, and unless surgical measures are adopted early the disease hastens to a fatal ending in almost every instance. Perforation of the stomach is usually described as being of two varieties, acute and chronic, but there is an intermediate class of cases, not embraced by either of these terms, which is best described as subacute. In acute perforation the ulcer gives way suddenly and completely. A larger or smaller hole results and through this the stomach contents are free to escape at once into the general cavity of the peritoneum. In subacute perforation the ulcer probably gives way almost as quickly as in the acute form, but owing to the small size of the ulcer, or to the emptiness of the stomach, or to the instant plugging of the opening by an omental flap or tag, or to the speedy formation of lymph which forms, as it were, a cork or lid for the ulcer, the escape of fluid from the stomach is small in quantity and the damage inflicted thereby is less considerable. The symptoms at their onset may be as grave as those in acute perforation, but on opening the abdomen the ulcer may be seen to be plugged and no further escape of fluid is occurring. In the subacute form of perforation $I$ have found that there is always a complaint of greater discomfort for several days preceding the rupture. Vague general or localised pains have been felt in the abdomen, or a sharp spasm or "stitch" when the patient turned quickly or attempted to laugh. One girl, a housemaid, felt the pain down her left side especially when reaching up to her work; another said that it hurt her to bend as her side felt stiff. These premonitory symptoms are important and if recognised they should enable us to take measures to prevent the occurrence of perforation. They doubtless have their origin in a localised peritonitis and the stiffness is due to the unconscious protection of an inflamed area by a muscular splint. In chronic perforation the ulcer has slowly eaten its way through the coats of the stomach and a protective peritonitis has had time to develop at the base. The escape of stomach contents is, therefore, merely local ; barriers of lymph confine the fluid to a restricted area and a perigastric abscess may form. A chronic perforation occurs more frequently on the posterior surface of the stomach and the epigastric abscess occasioned thereby is recognised as "subphrenic." The acute and subacute forms of perforating ulcer are more common on the anterior surface.

There can be no doubt that recovery by medicinal treatment alone is possible both in the acute and in the subacute forms of perforation. I have had two cases under my care in which a diagnosis of perforation had been made by competent medical men. In both an operation was impossible, as no skilled help was available until the urgency of the symptoms seemed to have passed off. When I operated many months later the evidences of peritonitis completely surrounding the stomach were undeniable. Though patients may recover their recovery cannot be urged as a reason for delay in withholding surgical help in all cases. For the possibility of spontaneous recovery, though not denied, is yet so remote as to make it imperative to adopt operative treatment at the earliest possible moment. The risk of operation is definite, the hazard of delay is immeasurable. There are times when the diagnosis may be difficult. If morphine has been administered to still the intolerable pain the patient's condition becomes placid and comfortable. It may be almost impossible then to recognise the extreme urgency of the.case. In such 